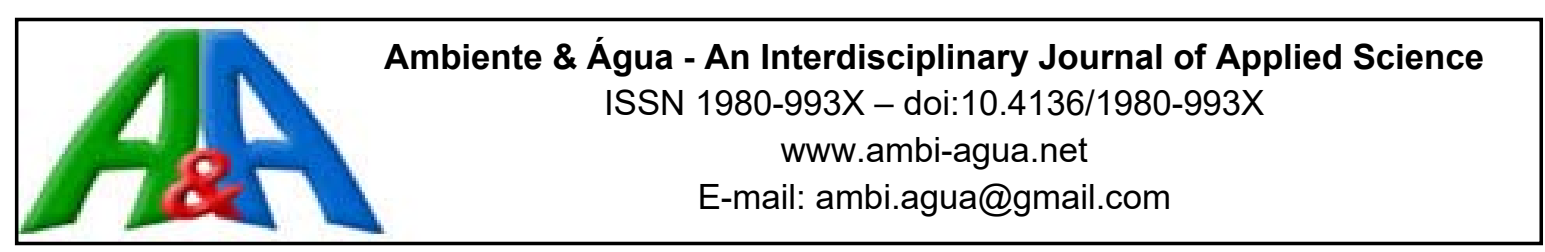

\title{
Calibração e validação do modelo SWAT para simulação hidrológica em uma bacia hidrográfica do litoral norte catarinense
}

\author{
doi:10.4136/ambi-agua.1951
}

Received: 1 Jun. 2016; Accepted: 18 Jan. 2017

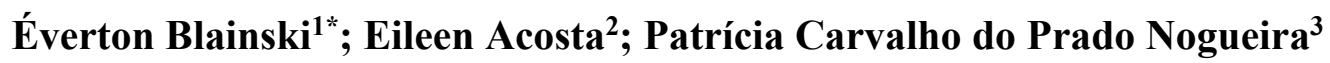 \\ ${ }^{1}$ Empresa de Pesquisa Agropecuária e Extensão Rural de SC (Epagri), Florianópolis, SC, Brasil \\ Centro de Informações de Recursos Ambientais e de Hidrometeorologia de SC (CIRAM) \\ ${ }^{2}$ The Nature Conservancy (TNC), Curitiba, PR, Brasil \\ Departamento de Ciências \\ ${ }^{3}$ Universidade Federal de Santa Catarina (UFSC), Florianópolis, SC, Brasil \\ Departamento de Engenharia Sanitária e Ambiental \\ *Autor correspondente: e-mail: evertonblainski@epagri.sc.gov.br, \\ eacosta@tnc.org, pati.cpn@hotmail.com
}

\section{RESUMO}

Os estudos hidrológicos em bacias experimentais têm sido realizados devido à necessidade de compreensão dos processos que controlam o movimento da água e sedimentos e seus impactos na disponibilidade e qualidade das águas. Os modelos hidrológicos são ferramentas com alto potencial para a simulação de cenários hipotéticos em diferentes escalas. Assim, o objetivo desse trabalho foi avaliar a eficácia do modelo SWAT para representação do fluxo hidrossedimentológico em uma bacia hidrográfica catarinense a partir dos procedimentos usuais de calibração e validação. O estudo foi realizado na bacia do rio Camboriú com uma área de $195 \mathrm{~km}^{2}$. Na análise do desempenho do modelo comparou-se os dados de vazão e sólidos transportados medidos e simulados na seção de controle. Os resultados obtidos atestaram a capacidade do SWAT para as simulações hidrossedimentológicas nessa bacia. Entretanto, foi preciso realizar a sua calibração para que as simulações atendessem os critérios de qualidade estabelecidos. Após a calibração, observou-se uma tendência de subestimativa das variáveis simuladas, porém, dentro de limites aceitáveis. Além disso, foi possível comprovar a eficiência do modelo para simulação das curvas de permanência das variáveis estudadas, caracterizando o SWAT como uma ferramenta importante na gestão de bacias hidrográficas como a do rio Camboriú.

Palavras-chave: gestão, transporte de sedimentos, vazão.

\section{Calibration and validation of the SWAT model for hydrologic simulation in a river basin located in the north coast of Santa Catarina}

\section{ABSTRACT}

Hydrological studies in experimental basins have been widely carried out in order to understand the processes that control the movement of water and sediment and their impact on the availability and quality of water. For this purpose, the hydrological models are used to simulate hypothetical scenarios at different spatial and temporal scales. The goal of this study was to evaluate the efficiency of the SWAT model to represent the hydrosedimentological flow 
in a Santa Catarina river basin by applying the usual procedures for calibration and validation. The study was conducted in the basin of the Camboriú River (BHRC), which has an area of 195 $\mathrm{km}^{2}$. For model performance analysis purposes, the flow and solids transported to the control section studied and the simulated data were compared. The results obtained have proven the efficiency of SWAT hydrosedimentological simulations in the BHRC. However, it was necessary to calibrate the model so that the simulations would comply with the established quality criteria. Though it occurred within acceptable limits, there was a tendency of underestimation of the simulated variables after calibration. Additionally, it was possible to verify the efficiency of the model for simulating the retention curves of the analyzed variables, thus emphasizing the SWAT as an important tool for river basin management such as the basin of Camboriú River.

Keywords: flow, management, sediment transport.

\section{INTRODUÇÃO}

Os estudos hidrológicos em bacias hidrográficas têm sido amplamente realizados a partir da necessidade de compreensão dos processos que controlam o movimento da água e sedimentos, e seus impactos sobre a disponibilidade e qualidade das águas. Nesse sentido, os modelos hidrológicos se consolidaram como ferramentas de representação desses processos com alto potencial para a simulação de cenários de mudanças climáticas, de alterações do uso do solo e de intervenções antrópicas em diferentes escalas espaciais e temporais.

A integração de modelos hidrológicos aos sistemas de informações geográficas proporcionou o desenvolvimento de ferramentas com maior embasamento físico, principalmente por possibilitar a representação dos parâmetros dos modelos de forma distribuída (Veith et al., 2010). Dessa integração, surgiu a possibilidade de ampliar o número de variáveis utilizadas para representar os fenômenos naturais dentro das bacias hidrográficas.

Apesar do grande número de variáveis incorporadas, os modelos hidrológicos são considerados representações simplificadas dos fenômenos naturais, e por mais sofisticados que sejam, ainda apresentam uma limitação relacionada à concepção e ao empirismo. De acordo com Muleta e Nicklow (2005), a qualidade dos resultados modelados depende da estrutura do modelo e dos algoritmos utilizados nas rotinas de cálculos, sendo que a qualidade dos dados de entrada para estimativa dos parâmetros representativos é de extrema importância.

Dentre as inúmeras opções de modelos hidrológicos disponíveis atualmente, o Soil and Water Assessment Tool (SWAT) tem sido bastante utilizado para simulações de vazões e sedimentos. O SWAT é um modelo semi-distribuído e contínuo no tempo, desenvolvido para simular o impacto da mudança do uso do solo em bacias hidrográficas de diferentes escalas (Arnold et al., 2012). Uma das facilidades para sua utilização é a interface acoplável aos principais softwares de geoprocessamento, o que possibilita a integração de bancos de dados, a elaboração e edição de cenários climatológicos e de uso das terras, e a análise e representação espacial das variáveis simuladas. No entanto, vale ressaltar que o SWAT é composto por um conjunto de equações que representam de forma simplificada o movimento da água, sedimentos e nutrientes em um sistema natural, razão pela qual deve ser calibrado e validado a partir de dados observados.

De acordo com Brighenti et al. (2016), é possível encontrar na literatura mais de 650 artigos publicados utilizando o SWAT como ferramenta de simulação. No Brasil, entre 1999 e 2013 foram publicados 102 trabalhos que relatam a utilização do SWAT para simulação do fluxo hidrossedimentológico. No entanto, um grande número dos trabalhos não descreve os processos de calibração e, principalmente, validação do modelo (Bressiani et al.,2015). Segundo os autores, a principal dificuldade para realizar a calibração e a validação de modelos é a ausência de séries de dados medidos nas bacias modeladas. 
A calibração do SWAT tem por objetivo principal minimizar os desvios entre as variáveis simuladas e medidas. Esse processo demanda amplo conhecimento em virtude do grande número de parâmetros não mensuráveis que precisam ser estimados (Veith et al., 2010). O processo de calibração automática facilita essa tarefa através da utilização de técnicas computacionais avançadas que possibilitam a alteração de parâmetros previamente selecionados dentro de uma faixa de valores estabelecida. A calibração pode ainda ser facilitada a partir da determinação do grau de influência dos parâmetros do modelo sobre a variável simulada. Para isso, a análise de sensibilidade possibilita identificar os parâmetros que mais influenciam no modelo e sua importância nos resultados gerados.

Por fim, a última etapa de verificação de um modelo hidrológico é a validação dos ajustes realizados nos parâmetros. A validação se resume em executar o modelo com os valores dos parâmetros determinados na calibração para um período de dados diferente ao utilizado na calibração (Arnold et al., 2012). Uma vez atendidos os critérios mínimos de qualidade, o modelo pode ser considerado adequado para a simulação do fluxo hidrossedimentológico em bacias experimentais.

Diante do potencial do SWAT como ferramenta de simulação e da carência de estudos aprofundados dessa ferramenta aplicada à pequenas bacias agrícolas no Brasil, buscou-se, com esse trabalho, aplicar a metodologia padrão de análise de sensibilidade, calibração e validação do SWAT visando avaliar sua eficácia para representação do fluxo hidrossedimentológico na bacia hidrográfica do rio Camboriú, Santa Catarina.

\section{MATERIAL E MÉTODOS}

\section{1. Área de estudo}

A bacia hidrográfica do rio Camboriú (BHRC) possui uma área de $195 \mathrm{~km}^{2}$ e abrange os municípios de Camboriú e Balneário Camboriú no estado de Santa Catarina (Figura 1). De acordo com a classificação de Köeppen, o clima dessa região é classificado como mesotérmico úmido subtropical, com temperatura média no mês mais frio inferior a $18^{\circ} \mathrm{C}$ e temperatura média no mês mais quente acima de $22^{\circ} \mathrm{C}$, com verões quentes e geadas pouco frequentes. A precipitação pluviométrica pode variar de $1.430 \mathrm{~mm}$ a $1.908 \mathrm{~mm}$ e existe a tendência de concentração das chuvas nos meses de verão, contudo sem estação seca definida.

Os solos predominantes da BHRC foram os Cambissolos, os Argissolos e os Gleissolos, ocupando, respectivamente, $48,7 \% ; 16,5 \%$ e $12,7 \%$ da área total da bacia. O mapeamento desses solos foi realizado com base em amostras coletadas em duas toposequências delimitadas ao longo da bacia. A partir da descrição dos perfis e dos resultados das análises físico-químicas, determinou-se os polígonos referentes às classes de solos com base na parametrização descrita em Embrapa (2013).

Entre as classes de uso e ocupação das terras, as áreas florestadas ocuparam $62,8 \%$ da área total da bacia, seguida pelas áreas com pastagem $(17,0 \%)$, áreas urbanas $(8,2 \%)$, arroz irrigado $(5,7 \%)$, reflorestamento $(2,5 \%)$, estradas rurais $(1,7 \%)$, solo exposto $(1,2 \%)$, massas d'água $(0,5 \%)$ e culturas anuais $(0,4 \%)$. O mapa de uso e ocupação foi gerado a partir de ortofotos correspondentes ao ano de 2012. A interpretação das imagens foi realizada através de ferramentas de processamento digital associada à coleta de dados utilizando GPS.

O modelo digital do terreno (MDT) foi obtido a partir do levantamento aerofotogramétrico de Santa Catarina realizado pela Secretaria de Desenvolvimento Sustentável (SDS/SC) no ano de 2010, adaptado para uma resolução espacial de 3 metros. O relevo da bacia hidrográfica foi dividido em cinco classes de declividade delimitadas com base no MDT e adaptadas à rotina de cálculos do modelo conforme classificação a seguir: 0 a 6\%; 6 a 20\%; 20 a 35\%; 35 a 48 e $>48 \%$.

Rev. Ambient. Água vol. 12 n. 2 Taubaté - Mar. / Apr. 2017 


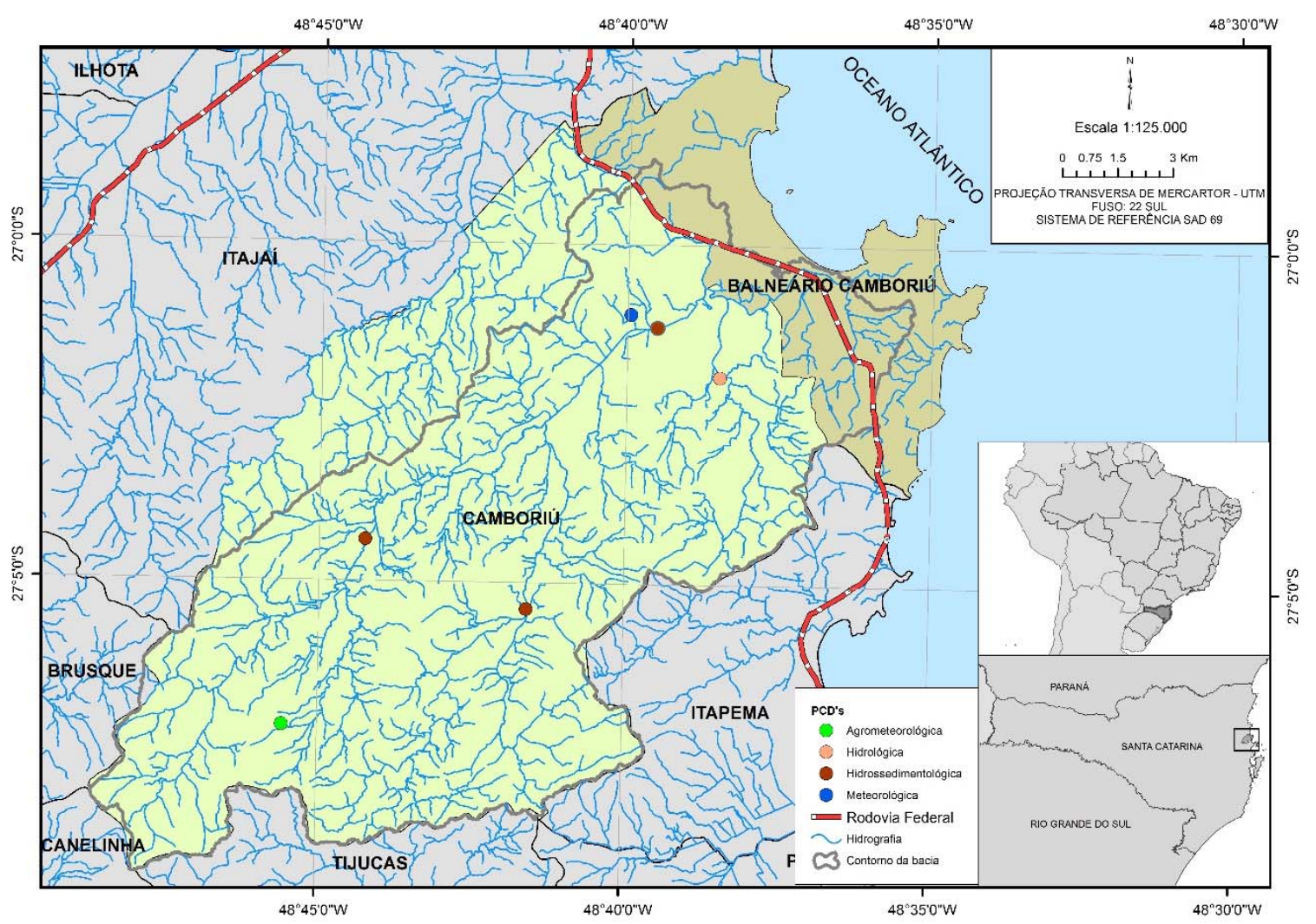

Figura 1. Mapa de localização da bacia hidrográfica do rio Camboriú.

\subsection{Modelo SWAT}

O SWAT é um modelo hidrológico fundamentado em características físicas de bacias hidrográficas, contínuo no tempo e com capacidade de simular longos períodos (Arnold et al., 2012). De acordo com os autores, o modelo foi desenvolvido com uma estrutura de comandos capaz de dividir a área em unidades homogêneas tendo como componentes aspectos climáticos, edáficos, topográficos, vegetativos e de manejo do solo, possibilitando a simulação de escoamento, sedimentos e nutrientes em bacias hidrográficas.

O componente hidrológico do modelo inclui sub-rotinas para determinação de escoamento superficial, percolação, fluxo lateral subsuperficial, fluxo de retorno do aquífero raso e evapotranspiração.

A perda de solo foi calculada a partir da Equação Universal de Perda de Solo Modificada - MUSLE (Williams, 1975). Essa metodologia utiliza a quantidade de escoamento para simular a erosão e a produção de sedimentos. A MUSLE é definida conforme Equação 1.

$$
\text { Sed }=11,8 \cdot\left(Q_{\text {surf }} \cdot q_{\text {peak }} \cdot \text { area }_{\text {hru }}\right)^{0,56} \cdot K_{U S L E} \cdot C_{U S L E} \cdot P_{U S L E} \cdot L S_{U S L E} \cdot C F R G
$$

em que:

Sed - perda do solo por unidade de área (ton);

Qsurf-é o volume de escoamento superficial $\left(\mathrm{mm} \mathrm{ha}^{-1}\right)$;

$q_{p e a k}$ - é o índice máximo de escoamento $\left(\mathrm{m}^{3} \mathrm{~s}^{-1}\right)$; area $a_{h r u}$-área de cada unidade de resposta hidrológica (ha);

$K_{U S L E}$ - fator de erodibilidade do solo;

CusLe - fator de uso e manejo;

PUSLE - fator práticas conservacionistas; 
$L S$ - fator topográfico; e

$C F R G$ - fator de fragmento bruto.

A vazão de pico foi calculada por uma modificação do Método Racional. O escoamento superficial foi obtido através do método Curva-Número do Soil Conservation Service (Neitsch et al., 2011).

O transporte de sedimentos através da rede de canais foi baseado nos processos de deposição e degradação. A deposição dentro do canal até o exutório da bacia foi determinada a partir da velocidade de deposição das partículas do sedimento (Melo Neto et al., 2014). A velocidade de sedimentação foi calculada a partir da lei de Stokes e a degradação do sedimento no canal foi fundamentada no conceito do poder de fluxo de Bagnold (1977).

A evapotranspiração potencial foi definida a partir do método de Penman-Monteith (Allen et al., 1989) e o ciclo hidrológico simulado pelo SWAT foi baseado na equação do balanço hídrico, conforme apresentado na Equação 2.

$$
S W_{t}=S W_{0}+\sum_{i=1}^{t}\left(R_{\text {day }}-Q_{\text {surf }}-E_{a}-W_{\text {seep }}-Q_{g w}\right)
$$

em que:

$$
\begin{aligned}
& S W_{t} \text { - conteúdo final de água no solo }(\mathrm{mm}) ; \\
& S W_{0} \text { - conteúdo de água no solo }(\mathrm{mm}) ; \\
& \mathrm{t} \text { - tempo (dias); } R_{\text {day }} \text { - precipitação }(\mathrm{mm}) ; \\
& Q_{\text {surf }} \text { - escoamento superficial }(\mathrm{mm}) ; \\
& E_{a} \text { - evapotranspiração }(\mathrm{mm}) ; \\
& W_{\text {seep }} \text { - percolação de água da camada simulada para a inferior }(\mathrm{mm}) ; \text { e } \\
& \text { Qgw }_{\mathrm{g}} \text { - escoamento lateral }(\mathrm{mm}) .
\end{aligned}
$$

\subsection{Dados hidrossedimentológicos}

Todos os dados hidrossedimentológicos foram coletados e transmitidos automaticamente a partir de plataformas de coleta de dados (PCD's) distribuídas ao longo da bacia hidrográfica (Figura 1).

No monitoramento meteorológico, a variável precipitação foi coletada nas seis PCD's (Figura 1). Já as variáveis temperatura e umidade relativa do ar foram registradas exclusivamente nas PCD's dos tipos meteorológica e agrometeorológica. Por fim, as demais variáveis (velocidade do vento e radiação solar) foram monitoradas somente na PCD meteorológica, conforme identificado na Figura 1. Todavia, a partir desses equipamentos registrou-se dados diários entre 2012 e 2015. A fim de ampliar a série de dados climatológicos, utilizou-se uma PCD instalada nas imediações da bacia hidrográfica. Assim, obteve-se um conjunto de dados compreendido entre os anos de 1981 e 2015.

$\mathrm{O}$ monitoramento hidrossedimentológico foi realizado a partir de sensores acoplados às PCD's do tipo hidrossedimentológicas (Figura 1). Nos três locais monitorados, determinou-se a vazão a partir do nível d'água associado às curvas-chave estabelecidas em campanhas de medição hidrológica. Ainda nesses pontos, foi determinado, indiretamente, o volume de sólidos transportados a partir do monitoramento contínuo de turbidez (NTU) e da correlação dessa variável com o conteúdo de sólidos suspensos total (SST) medido ao longo do período de 2012 a 2015. Por fim, a quantidade de sólidos transportados foi determinada pela multiplicação do conteúdo de SST pela vazão acumulada diariamente. 


\subsection{Calibração e validação do modelo}

A calibração do modelo SWAT foi realizada de maneira automatizada pelo software SWAT-CUP. Dentre as opções disponíveis no SWAT-CUP para os procedimentos de calibração e análise de incertezas, optou-se pelo método SUFI2 (Sequential Uncertainty Fitting). Nesse processo é utilizado o método de hipercubo latino para a definição dos parâmetros de análise, e o usuário define o intervalo para a calibração e o número de simulações a serem realizadas, a fim de definir o melhor ajuste (Abbaspour et al., 2007).

Para a etapa de calibração, foi selecionado o intervalo de dados diários de vazão e transporte de sedimentos compreendidos entre 01/01/2014 e 31/12/2014, enquanto que para a validação do ajuste do modelo foi selecionado o período de 01/01/2015 a 31/12/2015.

\subsection{Funções objetivo}

Para avaliar o desempenho do SWAT foram utilizadas as seguintes estatísticas de precisão: coeficiente de Nash-Sutcliffe (NSE), Equação 3, e o coeficiente de massa residual (CMR), descrito na Equação 4.

A correlação perfeita entre dados simulados e observados é expressa por um valor de $\mathrm{NSE}=1$ (Equação 3). Valores de NSE $<0$ demonstram que a utilização da média dos valores observados é melhor do que os resultados simulados (Brighenti et al., 2016).

$$
N S E=\left[\frac{\sum_{i=1}^{n}\left(Q_{i}^{o b s}-Q_{i}^{s i m}\right)^{2}}{\sum_{i=1}^{n}\left(Q_{i}^{\text {obs }}-Q^{\text {mean }}\right)^{2}}\right]
$$

em que:

NSE - coeficiente de Nash e Sutcliffe;

$n$ - número total de observações;

$Q_{i}{ }^{\text {obs }}$ - vazão observada;

$Q_{i}^{\text {sim }}$ - vazão simulada; e

$\mathrm{Q}^{\text {mean }}$ - média da vazão observada no período.

A tendência média entre valores simulados e medidos é expressa pelo CMR (Equação 4). $\mathrm{CRM}=0$ indica uma relação perfeita entre os dados, valores positivos indicam uma tendência de subestimativa do modelo, enquanto que valores negativos sinalizam para a superestimativa da variável simulada.

$$
C M R=\left[\frac{\sum_{i=1}^{n} Q_{i}^{s i m}-\sum_{i=1}^{n} Q_{i}^{o b s}}{\sum_{i=1}^{n} Q_{i}^{s i m}}\right]
$$

em que:

$C M R$ é o coeficiente de massa residual;

$Q_{i}{ }^{\text {sim }}$ é a vazão simulada pelo modelo; e

$Q_{i}{ }^{o b s}$ é a vazão medida.

Para classificação do desempenho do SWAT foi adotada a escala definida por Santhi et al. (2001): Muito bom: $N S E \geq 0,65$; Bom: 0,65 $>N S E \geq 0,54$; satisfatório: $0,54>N S E \geq 0,50$. Para o $C M R$ foi utilizada escala proposta por Liew et al. (2007): Muito bom: $|C M R| \leq 10 \%$; Bom: $10 \%<|C M R| \leq 15 \%$; Satisfatório: $15 \%<|C M R| \leq 25 \%$; Inadequado: $|C M R|>25 \%$. 


\section{RESULTADOS E DISCUSSÃO}

Na Tabela 1 estão listados os parâmetros classificados como mais sensíveis sobre as variáveis estudadas na escala temporal diária. Esses parâmetros foram identificados através da análise de sensibilidade realizada com o software SWAT-CUP. A lista completa dos parâmetros testados foi elaborada a partir de uma revisão bibliográfica em trabalhos que utilizaram os mesmos procedimentos.

Os parâmetros mostrados na Tabela 1 representam variáveis de difícil medição, ademais, existem poucas informações disponíveis acerca dessas variáveis para bacias hidrográficas brasileiras, principalmente para as de pequeno porte. Assim, o intervalo de valores adotado no processo de calibração foi definido com base em trabalhos dessa natureza (Santhi et al., 2001; Andrade et al., 2012; Arnold et al., 2012; Brighenti et al., 2016).

Tabela 1. Parâmetros utilizados na calibração do modelo SWAT com os valores ajustados e as respectivas faixas de variação.

\begin{tabular}{|c|c|c|c|c|}
\hline Ranking & Parâmetro & Descrição & $\begin{array}{l}\text { Valor } \\
\text { ajustado }\end{array}$ & $\begin{array}{l}\text { Faixa de } \\
\text { variação }\end{array}$ \\
\hline 1 & Lat_Time & Tempo necessário para o retorno do fluxo lateral (dias) & 42 & 0 a 180 \\
\hline 2 & Esco & $\begin{array}{l}\text { Fator de compensação da taxa de evaporação de água no } \\
\text { solo }\end{array}$ & 0,90 & 0 a 1 \\
\hline 3 & Gwqmin & $\begin{array}{l}\text { Nível limite da água no aquífero raso para a ocorrência } \\
\text { de fluxo de base }(\mathrm{mm})\end{array}$ & 1.000 & 0 a 5.000 \\
\hline 4 & Revapmn & $\begin{array}{l}\text { Profundidade limite de água no solo para a ocorrência } \\
\text { de ascensão da água à zona não saturada }(\mathrm{mm})\end{array}$ & 1.000 & 0 a 1.000 \\
\hline 5 & Alpha_BF & Constante de recessão do escoamento de base (dias) & 0,60 & 0 a 1 \\
\hline 6 & Gw_delay & $\begin{array}{l}\text { Intervalo de tempo necessário para a recarga do aquífero } \\
\text { (dias) }\end{array}$ & 0,65 & 0 a 500 \\
\hline
\end{tabular}

Uma etapa importante do processo de calibração e validação de um modelo hidrológico é a análise dos hidrogramas gerados e a comparação entre os resultados medidos e simulados. $\mathrm{Na}$ Figura 2, são apresentados os hidrogramas gerados nas fases de calibração e validação do SWAT. O período correspondente ao ano de 2014 foi selecionado para compor a etapa de calibração (Figura 2A), enquanto que o ano de 2015 foi utilizado para a validação do SWAT (Figura 2B). Em ambos os períodos, pode-se identificar uma aderência satisfatória entre os hidrogramas medidos e simulados.

As funções objetivo utilizadas para atestar a eficácia do SWAT corroboraram a sua eficiência para representar o fluxo hidrológico na BHRC. Na etapa de calibração, o NSE foi de 0,66 (Figura 2A). De acordo com a escala adotadas por Santhi et al. (2001) e com base nos valores de NSE, o modelo pode ser classificado como muito bom para a representação do fluxo de vazão na BHRC em escala de tempo diária. Na etapa de validação, o NSE foi de 0,89 (Figura 2B), ou seja, assim como na etapa de calibração, o modelo foi considerado muito bom para representação dessa variável (Santhi et al., 2001).

O CMR é a função objetivo que representa o percentual de viés entre dados simulados e medidos. Os resultados obtidos indicam um pequeno desvio em ambas as etapas. Na calibração, houve uma subestimativa de $3 \%$, e na fase de validação SWAT a subestimativa foi de $14 \%$ (Figura 2). De acordo com Liew et al. (2007) esses resultados indicam uma adequação muito boa do modelo SWAT para representação do fluxo hidrológico na primeira etapa e um desempenho bom do modelo na etapa de validação.

Com base nas funções objetivo utilizadas, pode-se concluir que o SWAT apresentou boa precisão nas estimativas de vazão representadas pelos valores de NSE. Além disso, foi possível constatar que as estimativas não apresentaram tendência significativa, dadas pelos baixos 
valores de CMR. Dessa forma, foi possível comprovar que os parâmetros foram devidamente ajustados à $\mathrm{BHRC}$ e não produziram estimativas com viés.

A quantidade de sólidos transportados diariamente ao longo da seção de controle é apresentada na Figura 3. Os sedimentogramas gerados na etapa de calibração (Figura 3A) e validação (Figura $3 \mathrm{~B}$ ) destacam o elevado grau de aderência entre as variáveis medidas e simuladas nos dois períodos. Na calibração, o NSE foi de 0,80 e o $C M R$ foi de 0,24 , ou seja, de acordo com Santhi et al. (2001), o SWAT foi classificado como muito bom para simulação da quantidade de sólidos transportada, entretanto, o CMR de 0,24 indica uma acentuada subestimativa da variável simulada. De acordo com a escala adotada por Liew et al. (2007), o modelo é classificado como satisfatório e com um viés de subestimativa da variável estudada, porém, dentro dos limites relatados na literatura. Vale destacar que devido a limitação de dados disponíveis de sólidos transportados, utilizou-se um período de nove meses na etapa de calibração. Além disso, observou-se que no período de calibração as vazões foram relativamente baixas, caracterizando um período de recessão, o que pode ter prejudicado a representação desta variável pelo SWAT.

Todavia, na etapa de validação, o desempenho do SWAT para simular a quantidade de sólidos transportada foi melhor do que na calibração, com NSE de 0,88 e CMR de 0,12. Tais resultados possibilitam classificar o SWAT como muito bom e com uma tendência de subestimativa de $12 \%$ da variável simulada, entretanto, com um viés não significativo.
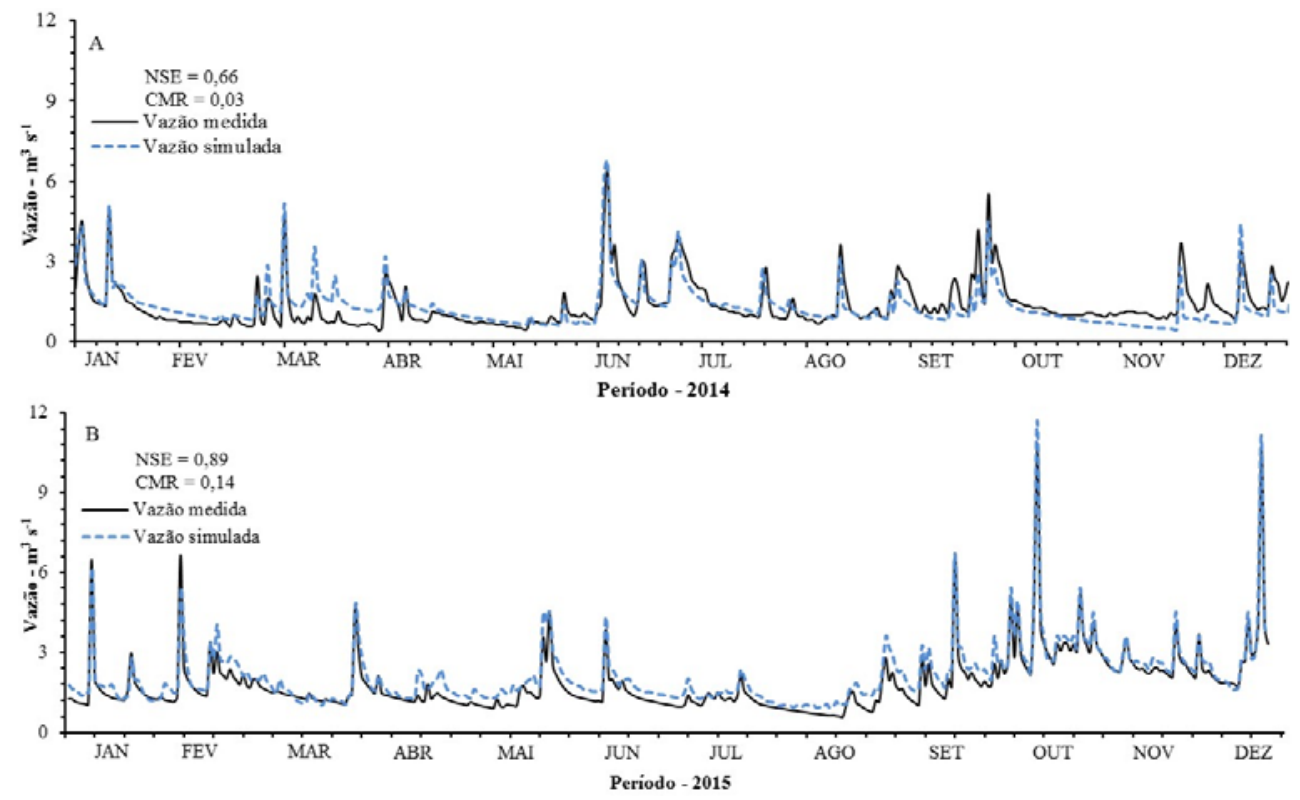

Figura 2. Hidrograma medido e simulado para a BHRC no período de calibração (A) e validação (B), na seção de controle.

Um aspecto importante associado à observação dos hidrogramas e sedimentogramas (Figuras 2 e 3) refere-se à dificuldade de simulação das vazões de cheia e, consequentemente, dos picos de sólidos transportados no período. Outros autores relataram as mesmas dificuldades e consideraram como aspectos limitantes a simplificação da distribuição das chuvas, o intervalo de simulação adotado na modelagem maior que o tempo de concentração das sub-bacias e o monitoramento hidrológico e climático com intervalo de coleta diário. Tais fatores, via de regra, limitam a resposta dos modelos às vazões máximas, dado ao reduzido tempo de concentração das sub-bacias (Bormann et al., 2007; Viola et al., 2009). 

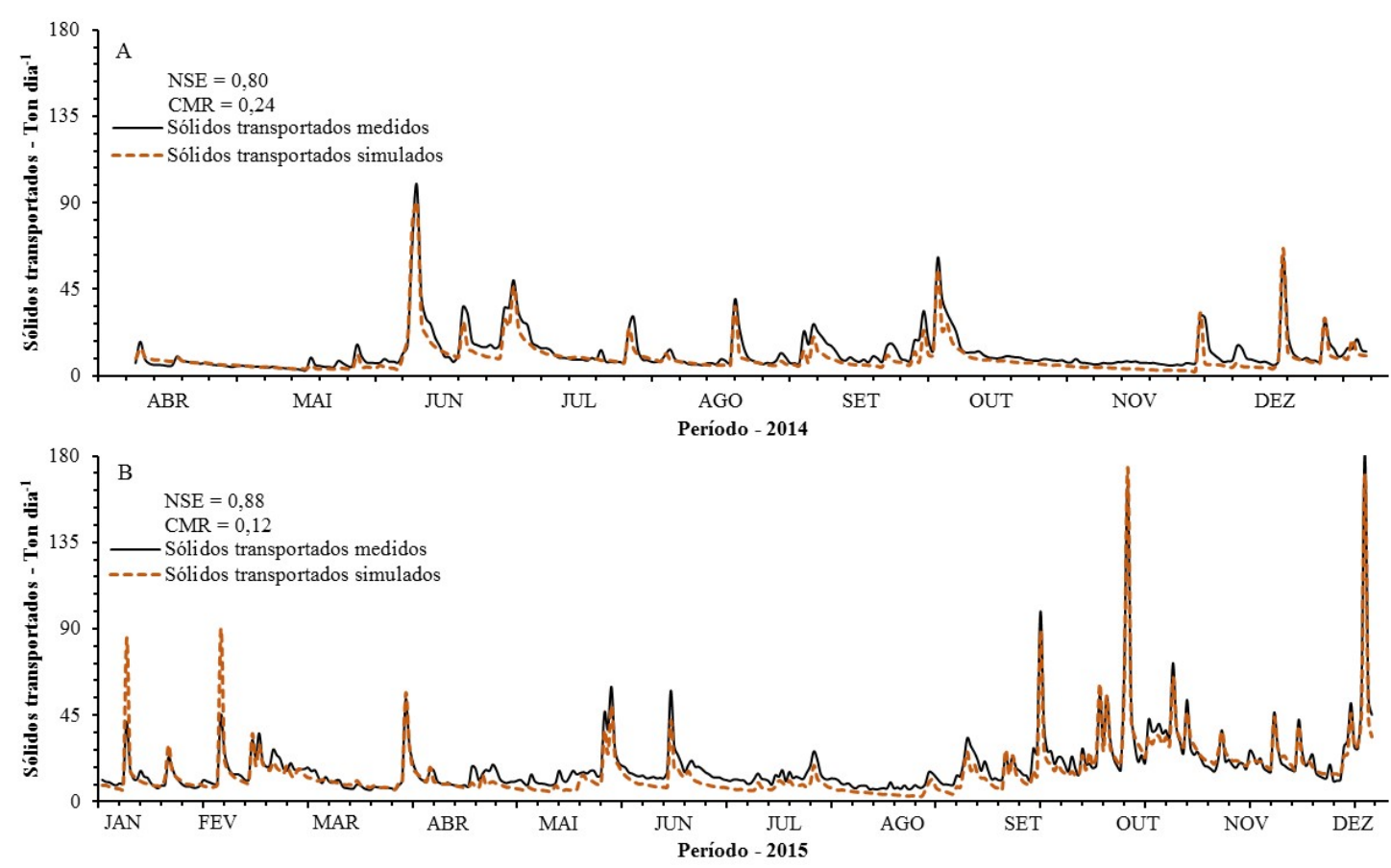

Figura 3. Sedimentograma medido e simulado para a BHRC no período de calibração (A) e validação (B) na seção de controle.

Entretanto, apesar da complexidade de simulações em bacia hidrográficas de pequeno porte e de algumas variações nas simulações dos picos de vazão e de sólidos transportados, verificou-se uma boa aderência dos hidrogramas e sedimentogramas simulados com relação aos medidos. Além disso, nos períodos de recessão, observou-se grande proximidade entre dados medidos e simulados, reforçando os resultados obtidos pelas funções objetivo.

Outra análise que pode ser feita com relação à calibração e validação de modelos hidrológicos diz respeito à capacidade de gerar séries de dados confiáveis para a elaboração das curvas de permanência. Essa funcionalidade dos modelos pode ser fundamental para a elaboração dos planos de bacias hidrográficas. Assim, os modelos hidrológicos podem ser úteis para uma gestão eficiente de recursos hídricos, principalmente em bacias hidrográficas de pequeno porte ou em regiões onde se tenham conflitos pelo uso da água.

De acordo com Viola et al. (2009), a partir de séries temporais de dados simulados pelos modelos hidrológicos, pode-se proceder a análise de vazões de referência como vazões de pico, de recessão e de referência para outorga d'água.

Para estimativa de vazões de referência para outorga há uma série de limitações para pequenas bacias, uma vez que é praticamente inexistente a disponibilidade de dados hidrológicos de vazão nesta escala no Brasil (Andrade et al., 2012). Nesses casos, as curvas de permanência geradas a partir de modelos hidrológicos constituem ferramentas importantes para a determinação da disponibilidade hídrica em bacias hidrográficas.

Na Figura 4 estão ilustradas as curvas de permanência observada e simulada pelo modelo para as variáveis vazão (Figura 4A) e sólidos transportados (Figura 4B). Cabe ressaltar que as curvas de permanência foram geradas com a série de dados utilizada na etapa de calibração e validação, ou seja, compreendem o período de 2014 a 2015.

A análise visual das curvas de permanência de vazão (Figura 4A) reforça o fato de ter ocorrido uma subestimativa de variável simulada. Todavia, o percentual de subestimativa esteve dentro de limites aceitáveis, principalmente para vazões de referência como a Q95\%, Q90\%, Q50\%, Q10\% e Q5\%, todas essas utilizadas como referência em projetos de hidráulica e em estudos relacionados aos recursos hídricos. Para as vazões de referência listadas 
anteriormente, os percentuais de subestimativa foram respectivamente de $16,9 \%, 14,8 \%$, $15,2 \%, 12,5 \%$ e $9,5 \%$.

Os resultados apresentaram o mesmo comportamento para a curva de permanência de sólidos transportados (Figura 4B), entretanto com magnitudes diferentes. Para valores com probabilidade de ocorrência de $95 \%$, o modelo subestimou a variável em $31,8 \%$, enquanto que para probabilidades de ocorrência menores como $10 \%$ ou $5 \%$, o modelo subestimou a variável simulada em $8,9 \%$ e $11,2 \%$ respectivamente, comparado ao valor medido.

De maneira geral, esses resultados indicaram que o SWAT apresentou uma tendência de subestimativa das variáveis simuladas, entretanto, dentro de limites aceitáveis dada a complexidade dos fenômenos envolvidos nos processos naturais que determinam o fluxo de água e sedimentos em bacias hidrográficas. Assim, ficou evidente o potencial do SWAT para simular de forma adequada as curvas de permanência na BHRC e demonstrando sua qualidade como ferramenta auxiliar na gestão dos recursos hídricos.

Com base em todas as análises e comparações realizadas entre os dados medidos e simulados pelo SWAT, foi possível constatar que o modelo apresentou alta precisão e adequação para simulação de vazões e de sólidos transportados em escala de tempo diária na BHRC. Ademais, foi possível verificar que os resultados dessa pesquisa estão de acordo com inúmeros trabalhos realizados no Brasil e no exterior, no tocante à utilização de modelos matemáticos para simulação do regime hidrossedimentológico em bacias hidrográficas experimentais.
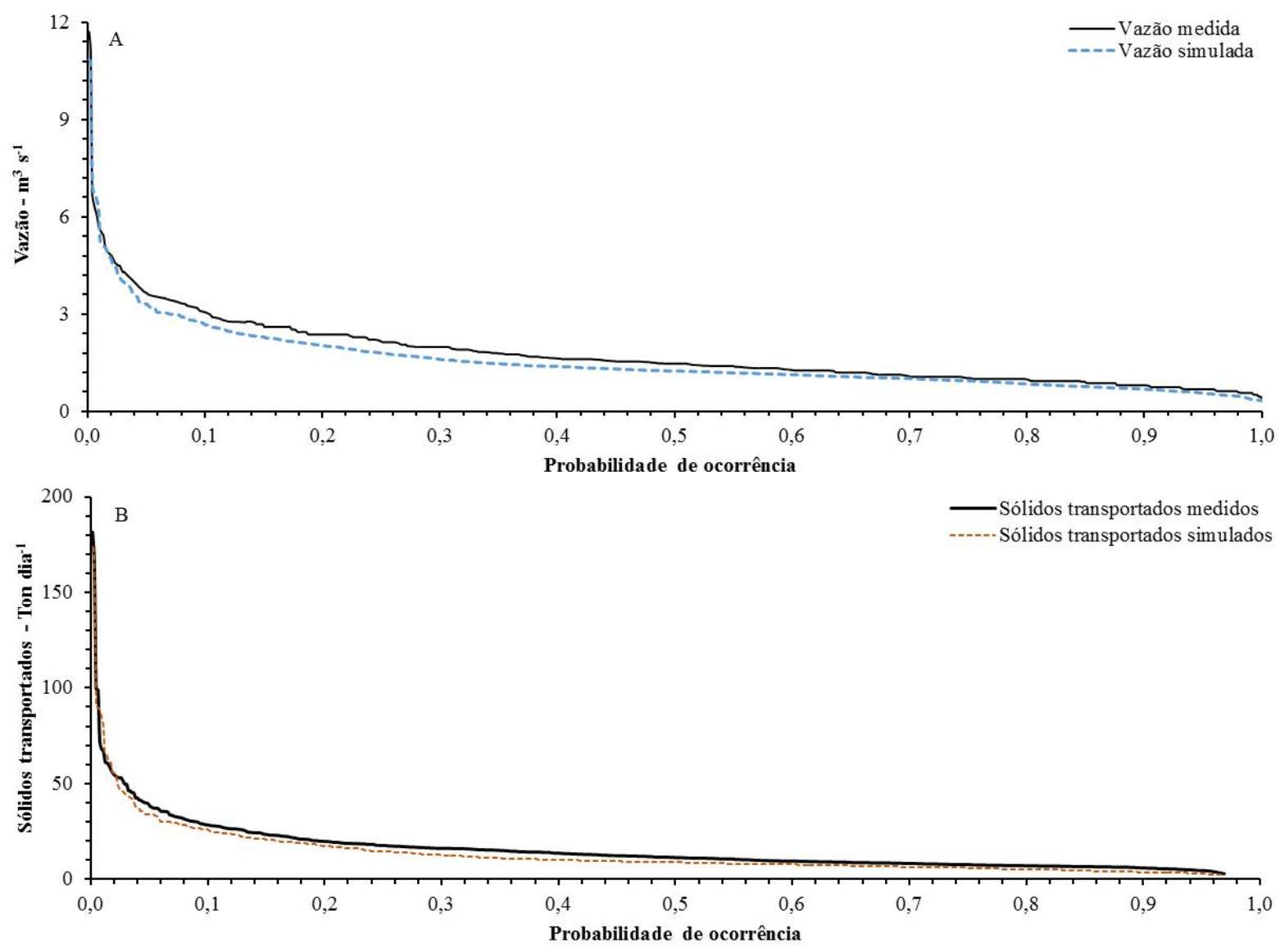

Figura 4. Curvas de permanência observadas e medidas para as variáveis vazão (A) e sólidos transportados (B) através seção controle instalada na BHRC. 


\section{CONCLUSÃO}

O modelo SWAT mostrou-se eficiente para as simulações hidrossedimentológicas na BHRC para a escala de tempo diária. Contudo, foi preciso realizar a sua calibração para que as simulações atendessem os critérios de qualidade estabelecidos.

Os resultados obtidos após a calibração do SWAT demonstraram uma tendência de subestimativa das variáveis simuladas, entretanto, dentro de limites aceitáveis, principalmente, devido à complexidade dos fenômenos envolvidos nos processos naturais que determinam o fluxo de água e sedimentos em bacias hidrográficas de pequeno porte.

Além dos resultados satisfatórios demonstrados pelas funções objetivo, foi possível atestar a eficiência do modelo para simulação das curvas de permanência das variáveis estudadas, caracterizando o SWAT como uma ferramenta importante para o processo de gestão de bacias hidrográficas como a do rio Camboriú.

\section{REFERÊNCIAS}

ABBASPOUR, K. C.; YANG, J.; MAXIMOV, I.; SIBER, R.; BOGNER, K.; MIELEITNER, J. et al. Modelling of hydrology and water quality in the pre-alpine/alpine Thur watershed using SWAT. Journal of Hydrology, v. 333, n. 2-4, p. 413-430, 2007. http://dx.doi.org/10.1016/j.jhydrol.2006.09.014

ALLEN, R. G.; JENSEN, J. L.; BURMAN, R. D. Operational Estimates of Reference Evapotranspiration. Agronomy Journal, v. 81, n. 4, p. 650-662, 1989. http://dx.doi.org/10.2134/agronj1989.00021962008100040019x

ANDRADE, M. A.; de MELO, C. R.; BESKOW, S. Simulação hidrológica em uma bacia hidrográfica representativa dos Latossolos na região Alto Rio Grande, MG. Revista Brasileira de Engenharia Agrícola e Ambiental, v. 17, n. 1, p. 69-76, 2012. http://dx.doi.org/10.1590/S1415-43662013000100010

ARNOLD, J. G.; MORIASI, D. N.; GASSMAN, P. W.; ABBASPOUR, K. C.; WHITE, M. J.; SRINIVASAN, R. et al. SWAT: Model use calibration and validation. Transactions of ASABE, v. 55, n. 4, p. 1494-1508, 2012.

BAGNOLD, R. A. Bed load transport by natural rivers. Water Resources Research, v. 13, n. 1, p. 303-312, 1977. http://dx.doi.org/10.1029/WR013i002p00303

BORMANN, H.; BREUER, L.; GRÄFF, T.; HUISMAN, J. A. Analyzing the effects of soil properties changes associated with land use changes on the simulated water balance: A comparison of three hydrological catchment models for scenario analysis. Ecological Modelling, v. 209, n. 1, p. 29-40, 2007.

http://dx.doi.org/10.1016/j.ecolmodel.2007.07.004

BRESSIANI, D. A.; GASSMAN, P. W.; FERNANDES, J. G.; GARBOSSA, L.; SRINIVASAN, R.; BONUMA, N. B. et al. A review of Soil and Water Assessment Tool (SWAT) applications in Brazil: challenges and prospects. International Journal of Agricultural and Biological Engineering, v. 8, n. 3, p. 1-27, 2015.

BRIGHENTI, T. M.; BONUMÁ, N. B.; CHAFFE, P. L. B. Calibração hierárquica do modelo Swat em uma bacia hidrográfica Catarinense. Revista Brasileira de Recursos Hídricos, v. 21, n. 1, p. 53-64, 2016. http://dx.doi.org/10.21168/rbrh.v21n1.p53-64 
EMPRESA BRASILEIRA DE PESQUISA AGROPECUÁRIA - EMBRAPA. Centro Nacional de Pesquisa de Solos. Sistema brasileiro de classificação de solos. 3 . ed. Brasília, 2013. 353 p.

LIEW, M. W.; VEITH, T. L.; BOSCH, D. D.; ARNOLD, J. G. Suitability of SWAT for the Conservation effects assessment project: A comparison on USDA-ARS watersheds. Journal of Hydrology Resources, v. 12, n. 2, p. 173-189, 2007. http://dx.doi.org/10.1061/(ASCE)1084-0699(2007)12:2(173)

MElO NETO, J. de O.; SILVA, A. M. da; MELlO, C. R. de; MELlO JÚNIOR, A. V. Simulação hidrológica escalar com o modelo SWAT. Revista Brasileira de Recursos Hídricos, v. 19, n. 1, p. 177-188, 2014. http://dx.doi.org/10.21168/rbrh.v19n1.p177-188

MULETA, M. K.; NICKLOW, J. W. Sensitivity and uncertainty analysis coupled with automatic calibration for a distributed watershed model. Journal of Hydrology, v. 306, p. 127-145, 2005. http://dx.doi.org/10.1016/j.jhydrol.2004.09.005

NEITSCH, S. L.; ARNOLD, J. G.; KINIRY, J. R.; WILLIAMS, J. R. Soil and Water Assessment Tool: Theoretical documentation - version 2009. Texas: Grassland, Soil and Water Research Laboratory - Agricultural Research Service; Blackland Research Center - Texas AgriLife Research, 2011.618 p.

SANTHI, C.; ARNOLD, J. G.; WILLIAMS, J. R.; DUGAS, W. A.; SRINIVASAN, R.; HAUCK, L. M. Validation of the SWAT model on a large river basin with point and nonpoint sources. Journal of American Water Resources Association, v. 37, n. 5, p. 1169-1188, 2001. http://dx.doi.org/10.1111/j.1752-1688.2001.tb03630.x

VEITH, T. L.; LIEW, M. W. VAN; BOSCH, D. D.; ARNOLD, J. G. Parameter sensitivity and uncertainty in SWAT: A comparison across five USDA-ARS watersheds. Transactions of ASABE, v. 53, p. 1477-1486, 2010.

VIOLA, M. R.; de MELlO, C. R.; ACERBI Jr., F. W.; DA SILVA, A. M. Modelagem hidrológica na bacia hidrográfica do Rio Aiuruoca, MG. Revista Brasileira de Engenharia Agrícola e Ambiental, v. 13, n. 5, p. 581-590, 2009. http://dx.doi.org/10.1590/S1415-43662009000500011

WILLIAMS, J. R. Sediment routing for agricultural watersheds. Water Resourses Bulletin, v. 11 n. 5, p. 965-974, 1975. http://dx.doi.org/10.1111/j.1752-1688.1975.tb01817.x 